\title{
Alignment of the High Beta Magnets in the RHIC Interaction Regions *
}

REOEIVED JUN 259997 D. Trbojevic, A. Jain, S. Tepikian, R. Grandinetti, G. Ganetis, J. Wei, and F. Karl, S T I
Brookhaven National Laboratory, Upton, NY, 11973, USA

\section{Abstract}

The betatron functions inside the triplet quadrupoles in the Relativistic Heavy Ion Collider-RHIC are of the order of $1500 \mathrm{~m}$, necessitating additional attention in the alignment procedure. On each side of the interaction regions eight cryogenic elements (six quadrupoles and two horizontal bending dipoles) are placed inside large cryostats. The quadrupole magnetic centers are obtained by "antenna" measurements with an accuracy of $\pm 60 \mu \mathrm{m}$. The signals from the "antenna" were cross calibrated with the colloidal cell measurements of the same magnet. The positions of the fiducials are related to the magnet centers during the "antenna" measurements. Elements are positioned warm inside the cryostats, with offsets to account for shrinkage during the cool down. The supports at the middle of the two central quadrupoles are fixed, while every other element slides longitudinally inside the cryostat during cool down or warm up.

\section{INTRODUCTION}

Two rings of the Relativistic Heavy Ion Collider cross each other in the horizontal plane at six interaction points. Twelve very large cryostats, located one on each side of the six interaction regions, house eight cryogenic elements- six quadrupoles and two dipoles. In the two interaction crossings (six o'clock and eight o'clock, at the RHIC detectors "STAR" and "PHENIX", respectively), the beams are focussed very tightly with the betatron functions of $\beta^{*}=1 \mathrm{~m}$. This is why special attention to the properties and alignment of the strong focusing quadrupoles, and dipoles close to them, is essential. Due to the large values of the amplitude betatron functions in these quadrupoles $(\sim 1500 \mathrm{~m})$ their influence on the beam properties is 5.5 times magnified with respect to the regular arc quadrupoles (where $\beta \sim 50 \mathrm{~m}$ ). There are three packages of multi-layer correction magnets between the triplet magnets. Two are directly connected to the $\mathrm{Q} 3$ while one is connected to the $\mathrm{Q} 2 \mathrm{mag}-$ net (see Figure 1). During production the magnetic centers of the correctors are measured and their positions optimized.

The magnetic field angles (rolls) are obtained from rotating coil measurements with an accuracy of $\pm 0.1 \mathrm{mrad}$. In addition to collisions of heavy ions, the RHIC accelerators will do spin physics experiments with two proton beams. The spin physics puts additional constraints on the alignment.

* Work performed under the auspices of the U.S. Department of Energy

\section{2 “COLD"AND "WARM" ELEMENT POSITIONS}

Positions of the supports or insulating posts of the large magnets inside the IP cryostats were established at room temperature. They remain unchanged with respect to the temperature conditions. Every magnet is installed at room temperature. Their longitudinal and vertical positions change during the cool-down when the temperature drops from $300 \mathrm{~K}$ down to the operating temperature of $4 \mathrm{~K}$.

\subsection{Vertical Shrinkage}

The vertical positions of the quadrupole and dipole centers were estimated to shrink for $\Delta l=2.743 \mathrm{~mm}$. The vertical shrinkage of the RHIC arc quadrupoles was estimated, by the same method, to be $\Delta l=1.905 \mathrm{~mm}$. Difference in the vertical positions of the RHIC arc quadrupole magnetic centers between room and $4 \mathrm{~K}$ temperature has been determined by four sets of measurements. The surveying instruments were used under the same conditions in both cases. It was important to allow 5 days cooldown period to establish correct and stable conditions. The value of $\Delta l=1.905$ $\mathrm{mm}$ was confirmed with an $\mathrm{rms}$ value of $\pm 38.1 \mu \mathrm{m}$.

\subsection{Longitudinal Positions}

The "D0-dipoles" are located at the end of the cryostat toward the interaction point. There are additional dipoles named as the "DX-dipoles", which belong to both rings. They bend one beam from the IP towards the D0 dipole and the other one from the D0 dipole towards the IP. The available aperture of the $\mathrm{DO}$ dipole is not larger than $\pm 6 \sigma$ of the beam. The DO dipole bends the beam either from the IP toward the DX magnet or it bends the beam from the DX magnet toward the triplet magnets. In the two interaction regions where the $\beta^{*}=1 \mathrm{~m}$ the amplitude betatron functions at the $\mathrm{D} 0$ dipoles are six times larger than at the regular bending dipoles in the RHIC arcs, although they are much shorter than the regular RHIC dipoles. The DO dipoles are only $3.6 \mathrm{~m}$ long.

The largest quadrupole in the IP region is the central " $Q 2$ " quadrupole. Every cryogenic element in the cryostat is supported by two posts. Their large "cold masses" are mechanically connected tightly to each other. The Q2 quadrupole cold mass has the side towards the IP fixed to the post below (see Figure 2). All other magnet supports are allowed to slide at the top of the cradles. A connection of the cold masses to the posts is provided by the cradles. All the cradles, with the exception of the one with the fixed position at the Q2 magnet, allow the cold masses to slide due to the shrinkage or expansion at lower or higher 


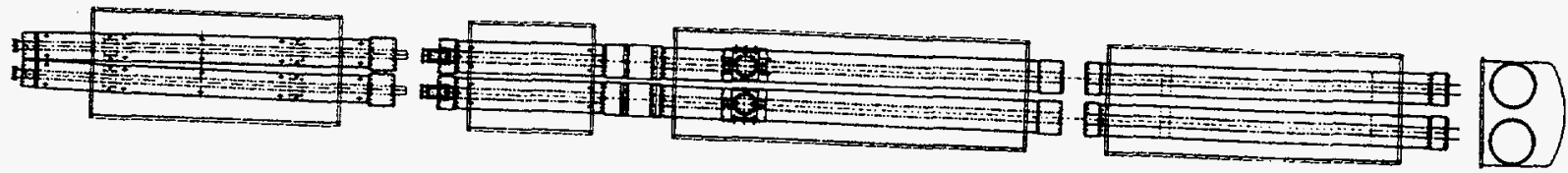

Figure 1: The layout of the high beta magnets.

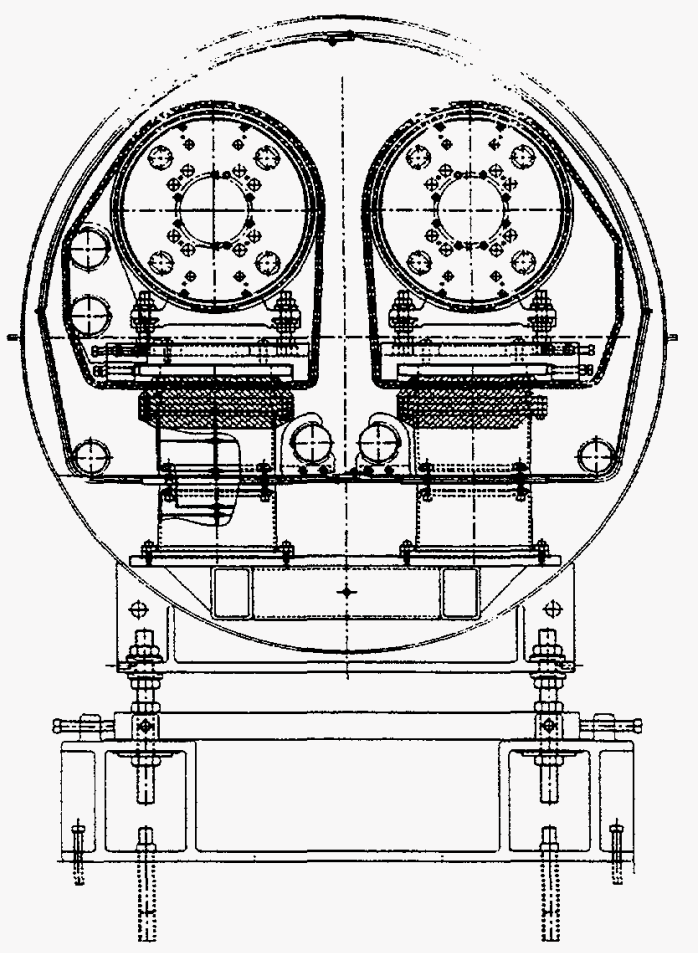

Figure 2: The cryostat cross section of the High Beta Magnets. The insulating posts and the stands are shown.

temperatures. Longitudinal positions of the cold masses at the posts' connections change as much as $22.7 \mathrm{~mm}$ at the magnet $\mathrm{Q} 3$, and $23.19 \mathrm{~mm}$ at the $\mathrm{D} 0$ dipole from the temperature difference between $300 \mathrm{~K}$ and $4 \mathrm{~K}$. A shrinkage constant of $\alpha=\left(L_{300 K}-L_{4 K}\right) / L_{300 K}=0.0031378$ has been experimentally established. The sliding was allowed with a special design of the cradles using the brass plates on both sides of the stainless steel blocks. A tolerance of $\pm 30 \mu \mathrm{m}$ between the block and the brass sides is achieved by two adjustable bolts on one side of the block. The DO magnet is a sector dipole with a curvature. The sagitta of the magnet is obtained from the mechanical measurements at five positions along the length. The holding blocks at the cradle positions of the $\mathrm{D} 0$ magnet were positioned with angles with respect to the straight beam line through the triplet magnets to get an optimum for the magnet position.

\section{MEASUREMENTS OF THE MAGNETIC CENTERS}

The colloidal cell magnetic measurements of the RHIC quadrupoles are reported elsewhere [1]. A survey antenna system also has been developed to measure the magnetic centers of quadrupoles as well as various corrector layers. The antenna has several harmonic windings to measure dominant harmonic and the feed down terms. Fiducials are placed on the antenna to locate the magnetic centers relative to the magnet fiducials. Magnetic centers are measured and electronically recorded in every RHIC quadrupole by either the "antenna" or colloidal cell measurements. Accuracy of the direct field center determination is within \pm $60 \mu \mathrm{m}$. Positions of the "antenna" coil or ferro-fluidic colloidal cell with respect to the outside fiducials are determined by the optical survey using a portable Leica ManCAT system with four theodolites. All data are transferred electronically and stored and used by the SYBASE data system. Only the best D0 dipoles and triplet magnets are assigned to the $\beta^{*}=1 \mathrm{~m}$ locations. In the process of magnet selection other important magnet properties like: alignment of the correctors, harmonic content, roll angles, quench performance, etc. are included. A prefiducialization of the high beta magnets is performed at the same magnet position. A relationship between the center of the magnetic field and the harmonic-antenna coil, or ferro-fluidic colloidal cell is obtained by using the ManCAT system at the same time and in the same local coordinate system.

\subsection{Location of the Beam Position Monitors}

There are two beam position monitors (BPM's) per ring (four inside the cryostat). One is located at the end of the quadrupole Q3, attached to the end plate. The other is between the D0 dipole and Q1 quadrupole attached to the end plate of the quadrupole Q1. A separate RF antenna system has been built for determining positions of the BPM's in the triplet magnets. A high frequency signal of $50 \mathrm{MHz}$ is sent to a rod placed close to the center of the BPM. Three positions of the rod with respect to the center of the BPM 
are used to accurately determine the position of the BPM's. The BPM plates provide signals which determine the position of the rod with respect to the plates. Part of the rod outside the triplet quadrupole cold mass vacuum flange has two fiducials. These are used to determine the rod position with respect to the outside cryostat fiducials by using the already mentioned ManCat system. The accuracy of the BPM position with respect to the outside fiducials is estimated to be $\pm 0.13 \mathrm{~mm}$. During the calibration procedure it was found that the reproducibility and accuracy of the measurements was $\pm 50 \mu \mathrm{m}$. The BPM positions are electronically transfered to the SYBASE and used by the high level BPM application code to correct for the real BPM positions.

\section{SURVEY AND ALIGNMENT}

The primary network of monuments in the tunnel had been established before [1]. The secondary network in the tunnel provides eight monuments on the floor, four on both sides of each triplet cryostat. Eight elements are brought to the tunnel after they had been presurveyed in the magnet production facility. They are positioned at the top of the posts with only the lower side of the cryostat built. The top of the cryostat, the bus connections, the 80K cryogenic shield, vacuum and beam pipe connections, etc. are built after the survey is completed.

The survey and alignment of the high beta elements were performed by using eight theodolites at the same time which are electronically connected to a Compaq 486c portable computer. Adjustments of the magnet positions are performed until the difference between the required and established positions in readings of at least four instruments are less than $\pm 25 \mu \mathrm{m}$.

\section{CONCLUSIONS}

A development of two new techniques, colloidal ferrofluidic cell and "antenna"measurements, allowed a very precise location of magnetic field centers within quadrupole magnets. Accuracy achieved in the center determination is $\pm 60 \mu \mathrm{m}$. Special attention to the presurvey, alignment and control of the roll angle of the high beta elements in RHIC is expected to provide better operation and higher luminosity of the colliding beams.

\section{ACKNOWLEDGEMENTS}

We would like to thank Mike Goldman who developed the ferrofluidic technique, Peter Cameron for developing the BPM antenna measurement system, Richard Thomas, Wing Louie, Andrew Marone for implementing measuring techniques, Mike Hemmer and Gerry Trahern for organizing the data analysis and transfer from the SYBASE to the ManCAT system, David McChesney for his contribution to the presurveying, James Cammarata and Sue Norton for providing the presented drawings, and the surveyors' teams who did a marvelous job surveying and aligning these elements.

\section{REFERENCES}

[1] D. Trbojevic, P. Cameron, G.L. Ganetis, M.A. Goldman, R. Gupta, M. Harrison, M.F. Hemmer, F.X. Karl, A. Jain, W. Louie, S. Mulhall, S. Peggs, S. Tepikian, R. Thomas, and P. Wanderer, "Alignment and Survey of the Elements in RHIC", Proceedings of the 1995 PAC and International Conference on High Energy Accelerators, held May 1-5, 1995, Dallas, Texas, TAA009,pp.2099-2101.

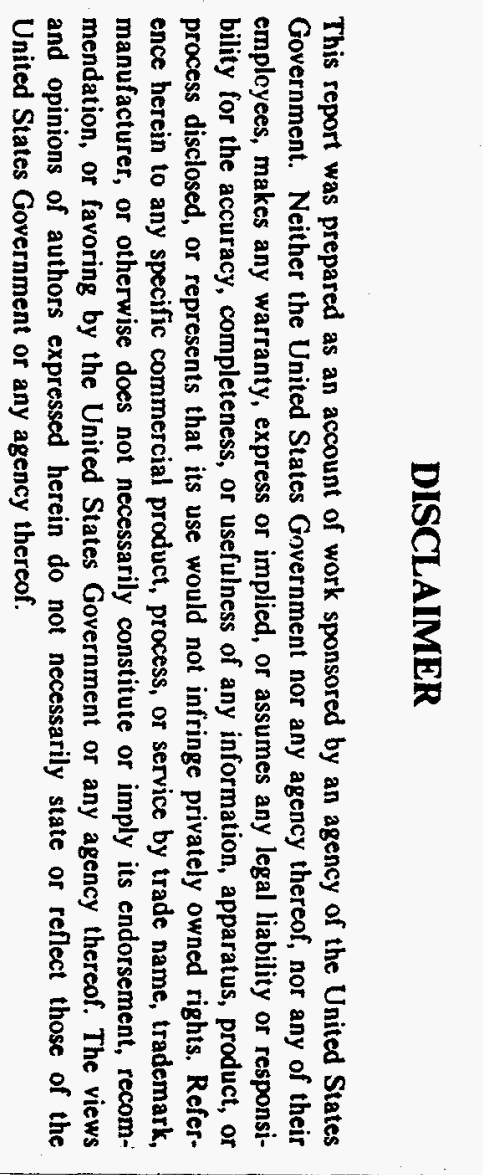




\section{DISCLAMIER}

Portions of this document may be illegible in electronic image produets. Images are produced from the best available original document. 